\section{BRAZIULIAN JOURNAL \\ OF MEDICAL AND BIOLOGICAL RESHARCH}

www.bjournal.com.br
ISSN 0100-879X

Volume 43 (12) 1135-1244 December 2010

BIOMEDICAL SCIENCES

AND

CLINICAL INVESTIGATION

Braz J Med Biol Res, December 2010, Volume 43(12) 1239-1244

doi: 10.1590/S0100-879X2010007500123

Intravenous regional block is similar to sympathetic ganglion block for pain management in patients

with complex regional pain syndrome type I

M.S.A. Nascimento, J.G. Klamt and W.A. Prado

The Brazilian Journal of Medical and Biological Research is partially financed by

Q

Ministério Conselho Nacional de Des
Cientifico e Tecrológico

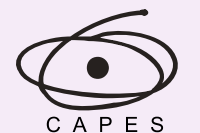

Ministério da Educação

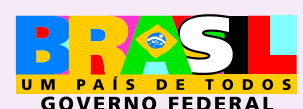

GOVERNO FEDERAL
DTAPESP

Institutional Sponsors

Hotsite of proteomics metabolomics developped by:

$$
\text { an }
$$

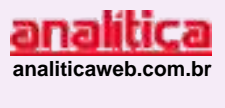




\title{
Intravenous regional block is similar to sympathetic ganglion block for pain management in patients with complex regional pain syndrome type I
}

\author{
M.S.A. Nascimento ${ }^{1}$, J.G. Klamt ${ }^{2}$ and W.A. Prado ${ }^{3}$ \\ ${ }^{1}$ Hospital do Câncer de Barretos, Fundação Pio XII, Unidade 2, Barretos, SP, Brasil \\ ${ }^{2}$ Departamento de Biomecânica, Medicina e Reabilitação do Aparelho Locomotor, \\ ${ }^{3}$ Departamento de Farmacologia, Faculdade de Medicina de Ribeirão Preto, \\ Universidade de São Paulo, Ribeirão Preto, SP, Brasil
}

\begin{abstract}
Sympathetic ganglion block (SGB) or intravenous regional block (IVRB) has been recommended for pain management in patients with complex regional pain syndrome type I (CRPS-I). Forty-five patients were initially selected but only 43 were accepted for the study. The present study evaluated the efficacy of IVRB produced by combining $70 \mathrm{mg}$ lidocaine with $30 \mu \mathrm{g}$ clonidine (14 patients, 1 male/13 females, age range: $27-50$ years) versus SGB produced by the injection of 70 mg lidocaine alone (14 patients, 1 male/13 females, age range: $27-54$ years) or combined with $30 \mu \mathrm{g}$ clonidine (15 patients, 1 male/ 14 females, age range: $25-50$ years) into the stellate ganglion for pain management in patients with upper extremity CRPS-I. Each procedure was repeated five times at 7-day intervals, and pain intensity and duration were measured using a visual analog scale immediately before each procedure. A progressive and significant reduction in pain scores and a significant increase in the duration of analgesia were observed in all groups following the first three blocks, but no further improvement was obtained following the last two blocks. Drowsiness, the most frequent side effect, and dry mouth occurred only in patients submitted to SGB with lidocaine combined with clonidine. The three methods were similar regarding changes in pain intensity and duration of analgesia. However, IVRB seems to be preferable to SGB due to its easier execution and lower risk of undesirable effects.
\end{abstract}

Key words: Clonidine; Complex regional pain syndrome type I; Intravenous regional block; Lidocaine; Stellate ganglion block; Sympathetic ganglion block

\section{Introduction}

Complex regional pain syndrome type I (CRPS-I), formerly known as reflex sympathetic dystrophy (RSD) is defined as "a syndrome that usually develops after an initiating noxious event, is not limited to the distribution of a single peripheral nerve, and is apparently disproportionate to the initiating event" (1).

Guidelines for pharmacological pain management of CRPS-I patients include oral use of tricyclic antidepressants, gabapentin, opioids, calcitonin, steroids, anticonvulsants, or antioxidants, most of which of limited efficacy (2). With persistent symptoms, sympathetic ganglion (SGB) or intravenous regional (IVRB) block may be recommended (2).

For several years SGB with a local anesthetic was considered to be a diagnostic test for the identification of CRPS-I patients, and the first choice for their management (3). A review of randomized controlled trials concluded that there is limited to no evidence that sympathetic blocks are effective in the treatment of CRPS-I (4). Nevertheless, SGB seems to be an effective approach for patients with CRPS-I (5-7), mainly when the procedure is initiated within 12 weeks of the onset of symptoms (8).

Intravenous regional anesthesia is considered to be a safe and effective way to provide anesthesia for short-duration hand surgery (9), and it can also contribute to the management of CRPS-I (10). However, the first publication on the usefulness of intravenous regional anesthesia for this purpose used guanethidine and was reported as IVRB (11). Conversely, IVRB with guanethidine in a trial of 57 CRPS-I patients was associated with more pain and vasomotor instability after 6 months (12). Other alternatives included IVRB with phentolamine, phenylepinephrine, reserpine, droperidol, or ketanserin but these agents were not more effective than a placebo (13).

IVRB with lidocaine alone failed to provide pain relief in

Correspondence: W.A. Prado, Departamento de Farmacologia, FMRP, USP, Av. Bandeirantes, 3900, 14049-900 Ribeirão Preto, SP, Brasil. Fax: +55-16-3633-2301. E-mail: wadprado@fmrp.usp.br

Received June 11, 2010. Accepted October 18, 2010. Available online November 5, 2010. Published December $20,2010$. 
CRPS-I patients beyond the duration of the block (14). Intravenous lidocaine was superior to a placebo in 16 patients with allodynia, but alleviation of spontaneous pain was achieved only with the highest dosage $(3 \mathrm{~g} / \mathrm{mL})$ and for a brief period of time (15). More recently, there was a report of a case of CRPS-I successfully treated with intravenous lidocaine alone (16).

Lidocaine combined with methylprednisolone has also been described for the treatment of post-traumatic dystrophy (17). In another randomized controlled trial, IVRB with methylprednisolone and lidocaine was not better than placebo in alleviating symptoms of CRPS-I (18). The combination of a local anesthetic with guanethidine, reserpine, bretylium, steroid, or ketorolac has been used for such purpose as well $(14,17,19,20)$. The addition of the $\alpha_{2}$-adrenergic agonist clonidine to lidocaine for intravenous regional anesthesia improved postoperative analgesia $(9,21)$ and produced complete pain relief in 5 of 7 patients with a diagnosis of CRPS-I of the knee (22). Successful treatment of RSD by IVRB with lidocaine and clonidine has been reported $(23,24)$. In our university hospital, IVRB with clonidine plus lidocaine has been routinely used for the management of CRPS-I (25). In contrast, the efficacy of lidocaine-induced SGB when combined with clonidine has not yet been reported.

The objective of the present study was to compare the efficacy of IVRB produced by combining lidocaine with clonidine, to that of SGB produced by the injection of lidocaine, alone or combined with clonidine, into the stellate ganglion, for the management of pain in patients with upper extremity CRPS-I. We found that the three procedures were equivalent in reducing pain intensity and increasing the duration of analgesia.

\section{Material and Methods}

The study was conducted on 45 outpatients referred to the Clinic for Pain Management of Hospital das Clínicas da Faculdade de Medicina de Ribeirão Preto. All patients had a diagnosis of upper extremity CRPS-I as established by the International Association for the Study of Pain (IASP) (1). After approval by the Committee on Human Research of the University Hospital, Faculty of Medicine of Ribeirão Preto, written informed consent was obtained from all patients before any procedural intervention. Utilization of placebo was not permitted by our institutional committee. All patients were evaluated at the first consultation regarding pain characteristics (localization, intensity, duration, quality, and irradiation) and skin temperature, and randomly assigned to one of three experimental groups of 15 patients each. They all reported unsuccessful use of tricyclic antidepressants, gabapentin, opioids, or anticonvulsants. At admission all patients declared to be free of drugs.

The second consultation was done 1 week later, when the patient was submitted to SGB with $70 \mathrm{mg} 1 \%$ lidocaine (Xylestesin ${ }^{\circledR}$ ) alone in group I (G-I) or combined with $30 \mu$ g clonidine in group II (G-II), or IVRB with 70 mg 1\% lidocaine combined with $30 \mu \mathrm{g}$ clonidine in group III (G-III). The drugs were purchased from Cristália Produtos Químicos e Farmacêuticos, Brazil, and the doses of lidocaine (26) and clonidine $(21,27)$ were within the range used in similar situations. The procedure was repeated once a week on four occasions. On all occasions, the intensity of pain was evaluated immediately before and soon after the end of each procedure using a $0-10-\mathrm{cm}$ visual analog scale (VAS: 0 = "no pain" and 10 = "worst pain imaginable"). One week after the last procedure, the intensity of pain was evaluated once again. After the end of each procedure, the patient remained in bed for up to 90 min to allow observation of any side effect due to block. Whatever the experimental group, blood pressure and heart rate were continuously recorded using a monitor apparatus (Dixtal Biomedica, Brazil), starting $15 \mathrm{~min}$ before and then up to $90 \mathrm{~min}$ after the SGB or IVRB procedure. During the blocking procedure, $\mathrm{O}_{2}$ saturation was also continuously monitored using the same apparatus. The difference $(\Delta)$ between the pain score obtained immediately before (iVAS) and after (fVAS) was calculated for each patient. A significant pain relief occurred whenever $\mathrm{NAS} \leq \mathrm{iVAS} / 2$ and $\Delta \geq 3$ were reported by the patient as suggested elsewhere (28). The patient was then asked to score pain intensity daily and record the time when VAS $\geq 3$ was perceived. The interval between this time and the end of each procedure was taken as the duration of analgesia.

A fluoroscopy-guided SGB was performed using the anterior paratracheal approach on the cervical sympathetic chain to reach the stellate ganglion under fluoroscopic view $(29,30)$. The skin temperature of the volar aspect of both hands under resting conditions was measured at room temperature $\left(22 \pm 2^{\circ} \mathrm{C}\right)$ before and after each blocking procedure using an electronic thermometer (Dixtal Biomedica). A 7.0-mL solution containing $70 \mathrm{mg} \mathrm{1 \%}$ lidocaine alone (G-I) or combined with $1 \mu \mathrm{g} / \mathrm{kg}$ clonidine (G-II) was used. A test volume of 0.5 to 1.0 $\mathrm{mL}$ was initially injected to exclude intravascular positioning, and the remaining volume was then administered. An increase of at least $2.2^{\circ} \mathrm{C}$ in the skin temperature of the affected limb over the temperature recorded before SGB was considered to be a successful block (30). The presence of Horner syndrome following SGB was not considered mandatory $(5,31)$.

IVRB was performed in patients of G-III as described elsewhere (32). A vein of the affected extremity was punctured, and the affected arm was exsanguinated by elevating its extremity for 1-2 min and wrapping it with an Esmarch bandage. A double tourniquet was then positioned around the upper affected arm and inflated to $50 \mathrm{mmHg}$ above the patient's systolic blood pressure. The bandages were then unwound and the arm was positioned horizontally. A 7.0-mL solution containing $1 \%$ lidocaine with $1 \mu \mathrm{g} / \mathrm{kg}$ clonidine was slowly injected through the punctured vein, and the tourniquet pressure was released 30 min later.

Patients were not included in the study if they had a history of cardiovascular disease, coagulopathy, neurologic degenerative disease or signs of sensory deficit caused by somatic peripheral block, cancer or diabetes, or were taking a monoaminoxidase inhibitor or an adrenergic or calcium antagonist. Patients were excluded from the study when a sign 
of hypersensitivity to lidocaine or clonidine was noticed or the total number of programmed blocks was not completed.

All procedures were conducted by one of the authors (MSDN). Side effects and effectiveness of treatment were recorded by another author (JGK) who was unaware of the procedure. The number of 15 patients per group was based on preliminary groups showing that the standard deviation of the mean VAS was less than 2. For a standard deviation of 2 , a sample size of 12 in each group has a $95 \%$ power to detect a difference between means of 3.09 with a level of significance ( $\alpha$ ) of 0.05 (twotailed), as determined using GraphPad StatMate 2 for Windows (GraphPad software, USA). The VAS scores reported immediately before each procedure and 1 week after the last procedure, and the duration of analgesia are shown as medians and interquartile ranges. The experimental groups were compared regarding VAS scores and duration of analgesia using the Kruskal-Wallis test. Post hoc comparisons were made using the Mann-Whitney U-test for differences between groups whenever the Kruskal-Wallis statistic was significant. Demographic data (age and gender) and temperature difference between left and right arms were compared by ANOVA followed by the Dunnett test. The level of significance was set at $\mathrm{P}<0.05$ in all cases.

\section{Results}

Two patients were excluded from the study due to an allergic reaction to lidocaine (one case in G-III) and an incomplete number of programmed blocks (one case in G-I). The demographic variables of the groups are shown in Table 1. There were no differences between groups regarding age, gender distribution or difference in skin temperature between the affected and the contralateral arm. The mean age of the patients was 38 years (range: $25-54$ years), and most of them were women (93\%). The most frequent causes of CRPS-I were repetitive strain injury $(\sim 42 \%)$, carpal tunnel syndrome ( 26\%), late post-surgical pain $(\sim 19 \%)$, and fracture followed by long-lasting immobilization ( $7 \%)$. Pain was more frequent in the right $(\sim 65 \%)$ than in the left arm. All patients fulfilled IASP criteria for CRPS-I diagnosis (1): they all had continuous pain that was accompanied by allodynia in 18 patients or hyperalgesia in 31 patients, reduced hand strength, skin discoloration, and changes in temperature perception. The signs and symptoms observed in the first consultation are also shown in Table 2. Edema (39 patients), tremors (28 cases) and sudomotor changes ( 25 cases) were frequently reported, but confirmed during clinical examination in only 23,13 , and 20 patients, respectively. The skin temperature in the affected arm was lower than in the contralateral arm in 36 patients. In contrast,

Table 1. Demographic characteristics of the experimental groups.

\begin{tabular}{lccc}
\hline & G-I & G-II & G-III \\
\hline Gender (male/female) & $1 / 13$ & $1 / 14$ & $1 / 13$ \\
Age range (years) & $27-54$ & $25-50$ & $27-50$ \\
Mean \pm SEM & $37.7 \pm 7.7$ & $38.6 \pm 7.0$ & $39 \pm 6.2$ \\
Affected arm (right/left) & $9 / 5$ & $10 / 5$ & $9 / 5$ \\
Causes & & & \\
$\quad$ Repetitive strain injury & 6 & 6 & 6 \\
Carpal tunnel syndrome & 2 & 4 & 5 \\
Late post-surgical pain & 4 & 2 & 2 \\
Fracture + long-lasting immobilization & 2 & 1 & 0 \\
$\quad$ Stab wound & 0 & 1 & 1 \\
$\quad$ Unknown origin & 0 & 1 & 0 \\
Disease duration range (months) & $3-72$ & $8-72$ & $2-48$ \\
Mean \pm SEM & $24.2 \pm 4.9$ & $24.2 \pm 4.2$ & $22.3 \pm 3.4$ \\
\hline
\end{tabular}

G-I = patients scheduled for stellate ganglion block with $70 \mathrm{mg}$ lidocaine; G-II $=$ patients scheduled for stellate ganglion block with $70 \mathrm{mg}$ lidocaine $+30 \mu \mathrm{g}$ clonidine; G-III = patients scheduled for intravenous regional block with $70 \mathrm{mg}$ lidocaine $+30 \mu \mathrm{g}$ clonidine.

Table 2. Signs and symptoms at the first consultation.

\begin{tabular}{lccc}
\hline & G-I & G-II & G-III \\
\hline Symptoms & & & \\
Continuous pain & 14 & 15 & 14 \\
Reduced hand strength & 14 & 15 & 14 \\
Skin discoloration & 14 & 15 & 14 \\
Perception of temperature change & 14 & 15 & 14 \\
Edema & 11 & 14 & 14 \\
Sudomotor changes & 3 & 12 & 10 \\
Tremors & 14 & 6 & 8 \\
Signs & & & \\
Allodynia & 4 & 7 & 7 \\
Hyperalgesia & 9 & 13 & 9 \\
Skin blood flow changes & 7 & 9 & 8 \\
Increased sudoresis & 2 & 7 & 7 \\
Reduced sudoresis & 1 & 1 & 2 \\
Edema & 7 & 7 & 9 \\
Tremors & 4 & 6 & 3 \\
Trophic changes & 7 & 11 & 11 \\
Reduced hand grip strength & 14 & 12 & 12 \\
Mean ( \pm SEM) temperature difference & $-2.1 \pm 0.4$ & $-2.5 \pm 0.3$ & $-1.7 \pm 0.4$ \\
\hline
\end{tabular}

Data are reported as number of patients. a Difference in skin temperature $\left({ }^{\circ} \mathrm{C}\right)$ between the affected arm and contralateral arm. G-I, G-II and G-III are identified in the legend to Table 1. 

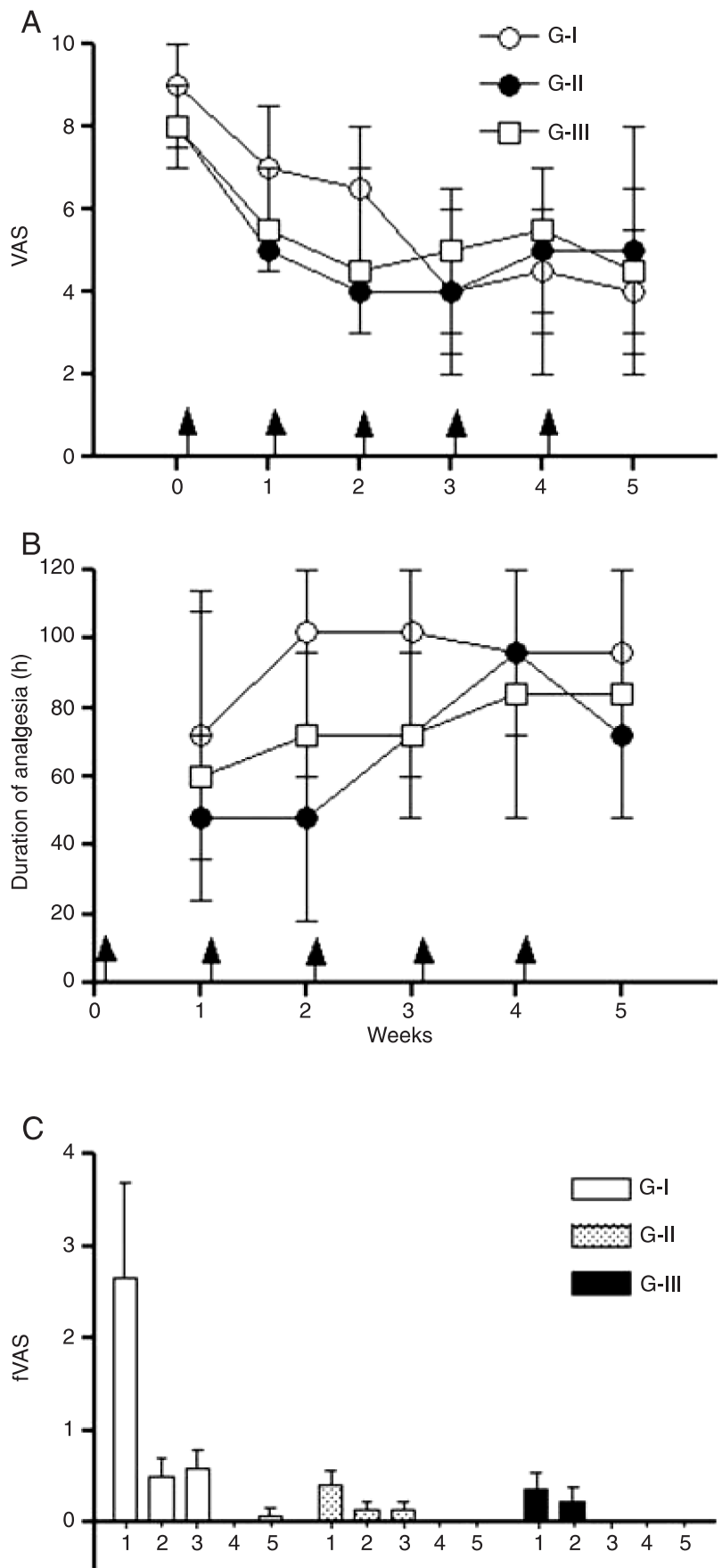

Figure 1. Time course of the changes in pain intensity $(A)$ and duration of analgesia (B) of patients with upper extremity complex regional pain syndrome type I submitted to stellate ganglion block with $70 \mathrm{mg}$ lidocaine alone (G-I, $\mathrm{N}=14)$ or with $30 \mu \mathrm{g}$ clonidine ( $\mathrm{G}-\mathrm{II}, \mathrm{N}=15)$, or intravenous regional block with lidocaine combined with clonidine (G-III, $\mathrm{N}=14)$. Each procedure was performed at the times indicated by the arrows. Pain intensity was assessed using a visual analog scale (VAS). Points are medians and interquartile ranges. The pain scores achieved soon after each procedure (fVAS) are shown in Figure C. the skin temperature in the affected arm was higher than in the contralateral arm in 2 patients.

The groups did not differ significantly $\left(F_{2,44}=1.28\right.$; $P=$ 0.22 ) regarding VAS values reported in the first consultation immediately before each procedure (mean \pm SEM: G-I = $8.7 \pm 0.3$; G-II = $7.9 \pm 0.4$; G-III = $8.3 \pm 0.3$ ). All patients had a significant reduction in VAS soon after the end of each procedure, but effective pain relief lasted less than 1 week in the majority of cases. The time-course of the VAS reported by the patients immediately before each procedure did not differ significantly between the experimental groups $\left(\mathrm{X}^{2} \leq\right.$ 5.52; $P \geq 0.06$, Kruskal-Wallis test). The VAS score was progressively reduced by all procedures from the first to the third block, but the remaining blocks did not produce a further decrease in VAS score (Figure 1A). The groups also did not differ concerning the duration of analgesia reported after each procedure $\left(X^{2} \leq 5.32 ; P \geq 0.069\right.$, Kruskal-Wallis test). Effective pain relief lasted progressively longer after the first to the third block, but the remaining blocks did not produce a further increase in the duration of analgesia (Figure 1B). The fVAS reported by the patients soon after the end of each procedure is shown in Figure $1 \mathrm{C}$.

Slight and nonsignificant changes in blood pressure and heart rate occurred in all groups regarding records obtained 15 min before and 90 min after each procedure (data not shown). The side effects observed in each group are summarized in Table 3. Drowsiness, the most frequent side effect, and dry mouth occurred only in patients submitted to SGB with lidocaine combined with clonidine. Less frequent side effects were nausea, dizziness, hoarseness, and pain at the puncture site.

\section{Discussion}

All patients selected for this study fulfilled IASP criteria for the diagnosis of CRPS-I, i.e., they had: 1) continuous pain, allodynia, or hyperalgesia disproportionate to the injury; 2) evidence at some time of edema, changes in skin blood flow, or abnormal sudomotor activity in the region of pain, and 3) no other conditions that would otherwise account

Table 3. Number of patients reporting side effects within $90 \mathrm{~min}$ following the analgesic procedure.

\begin{tabular}{lccc}
\hline & G-I & G-II & G-III \\
\hline Drowsiness & 0 & 14 & 6 \\
Nausea & 1 & 0 & 0 \\
Dizziness & 2 & 2 & 1 \\
Hoarseness & 1 & 2 & 0 \\
Pain at the site of injection & 1 & 1 & 0 \\
Dry mouth & 0 & 4 & 0 \\
\hline
\end{tabular}

G-I, G-II and G-III are identified in the legend to Table 1. 
for the degree of pain and dysfunction (1).

The present study demonstrates that SGB by lidocaine, with or without clonidine, or IVRB by lidocaine with clonidine led to a significant and comparable reduction in pain in patients suffering from CRPS-I in the upper arm. All procedures used here led to a progressive decrease in VAS scores and increased duration of analgesia following weekly repeated blocks for up to three trials. Further blocks, however, did not produce a further reduction in VAS scores or additional prolongation of the duration of analgesia. Therefore, improvement of the analgesic efficacy of either SGB or IVRB cannot be achieved by simply increasing the number of trials using any of the procedures studied.

Immediately after the end of the injection, all patients submitted to SGB had significant pain relief, as evaluated using a criterion described elsewhere (28). This result differs from that obtained in a recent report showing that only $40 \%$ of the patients with CRPS-I of the hands had complete pain relief following SGB with $5 \mathrm{~mL} 0.5 \%$ lidocaine, i.e., $25 \mathrm{mg}$ of local anesthetic (8). The higher dose of lidocaine used in the present study $(70 \mathrm{mg})$ may account for the difference.

Clonidine lacks analgesic properties when used alone for peripheral nerve block (33), but is frequently used to prolong the duration of local anesthesia in peripheral nerves or intravenous regional, epidural or intraspinal block procedures (34). The combination of clonidine with lidocaine improves postoperative analgesia and prolongs tourniquet tolerance during intravenous regional anesthesia (35). In the present study, SGB with lidocaine alone or combined with clonidine did not differ regarding effectiveness for pain management in CRPS-I patients. IVRB with lidocaine alone failed to provide pain relief in CRPS-I patients beyond the duration of the block $(14,15)$. However, an isolated case of CRPS-I treated successfully with only intravenous lidocaine has

\section{References}

1. Merskey H, Bogduk N. Classification of chronic pain. Seattle: IASP Press; 1994.

2. Quisel A, Gill JM, Witherell P. Complex regional pain syndrome: which treatments show promise? J Fam Pract 2005; 54: 599-603.

3. Hord ED, Oaklander AL. Complex regional pain syndrome: a review of evidence-supported treatment options. Curr Pain Headache Rep 2003; 7: 188-196.

4. Forouzanfar T, Koke AJ, van Kleef M, Weber WE. Treatment of complex regional pain syndrome type I. Eur J Pain 2002; 6: 105-122.

5. Price DD, Long S, Wilsey B, Rafii A. Analysis of peak magnitude and duration of analgesia produced by local anesthetics injected into sympathetic ganglia of complex regional pain syndrome patients. Clin J Pain 1998; 14: 216-226.

6. Karakurum G, Pirbudak L, Oner U, Gulec A, Karadasli H, Satana T. Sympathetic blockade and amitriptyline in the treatment of reflex sympathetic dystrophy. Int J Clin Pract 2003; 57: 585-587. been reported (16). In contrast, IVRB by lidocaine combined with clonidine has been successfully used earlier in CRPS-I patients $(22,36)$. The addition of clonidine to local anesthetics prolongs local anesthetic blocks (34), the mechanism of prolongation being at least in part pharmacokinetic (37). Alternatively, a hyperpolarization-activated cation current channel blockade may also contribute to enhancement of the lidocaine effect by clonidine (38).

The dose of clonidine used here appears to be well tolerated but drowsiness was often reported and was more frequent in patients submitted to SGB. The sedative effect of clonidine that usually occurs independent of the route of administration (34) may account for this effect. In addition to sedation, the major adverse effect of clonidine is dry mouth (39), but this effect occurred only in 4 patients submitted to SGB with combined lidocaine and clonidine. IVRB produced side effects in very few cases.

The effects of SGB with lidocaine, alone or combined with clonidine, or IVRB with lidocaine combined with clonidine, repeated weekly on five occasions, did not differ significantly regarding the intensity of pain or duration of analgesia in CRPS-I patients. Risks and contraindications of the use of IVRB are negligible, whereas side effects that may occur during stellate ganglion block include seizures, death, pneumothorax, and paralysis of the recurrent laryngeal nerve or brachial plexus (8). SGB may be preferred by skilful professionals regarding cost-effectiveness and time. If such professionals are not available, we conclude that IVRB is preferable to the SGB for management of CRPS-I due to its easier execution and lower risk of undesirable side effects. This notion reinforces a similar conclusion regarding the comparison of IVRB using guanethidine versus SGB using lidocaine for pain management of patients with RSD (40).
7. Wasner G, Schattschneider J, Binder A, Baron R. Complex regional pain syndrome - diagnostic, mechanisms, CNS involvement and therapy. Spinal Cord 2003; 41: 61-75.

8. Ackerman WE, Zhang JM. Efficacy of stellate ganglion blockade for the management of type 1 complex regional pain syndrome. South Med J 2006; 99: 1084-1088.

9. Reuben SS, Steinberg RB, Klatt JL, Klatt ML. Intravenous regional anesthesia using lidocaine and clonidine. Anesthesiology 1999; 91: 654-658.

10. Lake AP. Intravenous regional sympathetic block: past, present and future? Pain Res Manag 2004; 9: 35-37.

11. Hannington-Kiff JG. Relief of Sudeck's atrophy by regional intravenous guanethidine. Lancet 1977; 1: 1132-1133.

12. Livingstone JA, Atkins RM. Intravenous regional guanethidine blockade in the treatment of post-traumatic complex regional pain syndrome type 1 (algodystrophy) of the hand. J Bone Joint Surg Br 2002; 84: 380-386.

13. Harden RN. Pharmacotherapy of complex regional pain syndrome. Am J Phys Med Rehabil 2005; 84: S17-S28. 
14. McKain CW, Urban BJ, Goldner JL. The effects of intravenous regional guanethidine and reserpine. A controlled study. J Bone Joint Surg Am 1983; 65: 808-811.

15. Wallace MS, Ridgeway BM, Leung AY, Gerayli A, Yaksh TL. Concentration-effect relationship of intravenous lidocaine on the allodynia of complex regional pain syndrome types I and II. Anesthesiology 2000; 92: 75-83.

16. Toda K, Muneshige H, Asou T. Intravenous regional block with lidocaine for treatment of complex regional pain syndrome. Clin J Pain 2006; 22: 222-224.

17. Tountas AA, Noguchi A. Treatment of posttraumatic reflex sympathetic dystrophy syndrome (RSDS) with intravenous blocks of a mixture of corticosteroid and lidocaine: a retrospective review of 17 consecutive cases. $J$ Orthop Trauma 1991; 5: 412-419.

18. Taskaynatan MA, Ozgul A, Tan AK, Dincer K, Kalyon TA. Bier block with methylprednisolone and lidocaine in CRPS type I: a randomized, double-blinded, placebo-controlled study. Reg Anesth Pain Med 2004; 29: 408-412.

19. Hord AH, Rooks MD, Stephens BO, Rogers HG, Fleming LL. Intravenous regional bretylium and lidocaine for treatment of reflex sympathetic dystrophy: a randomized, double-blind study. Anesth Analg 1992; 74: 818-821.

20. Connelly NR, Reuben S, Brull SJ. Intravenous regional anesthesia with ketorolac-lidocaine for the management of sympathetically-mediated pain. Yale J Biol Med 1995; 68: 95-99.

21. Singelyn FJ, Gouverneur JM, Robert A. A minimum dose of clonidine added to mepivacaine prolongs the duration of anesthesia and analgesia after axillary brachial plexus block. Anesth Analg 1996; 83: 1046-1050.

22. Reuben SS, Sklar J. Intravenous regional anesthesia with clonidine in the management of complex regional pain syndrome of the knee. J Clin Anesth 2002; 14: 87-91.

23. Gintautas J, Housny W, Kraynack BJ. Successful treatment of reflex sympathetic dystrophy by Bier block with lidocaine and clonidine. Proc West Pharmacol Soc 1999; 42: 101.

24. Reuben SS. Preventing the development of complex regional pain syndrome after surgery. Anesthesiology 2004; 101: 1215-1224.

25. Frade LC, Lauretti GR, Lima IC, Pereira NL. The antinociceptive effect of local or systemic parecoxib combined with lidocaine/clonidine intravenous regional analgesia for complex regional pain syndrome type I in the arm. Anesth Analg 2005; 101: 807-811.

26. Brown DL. Somatic or sympathetic block for reflex sympathetic dystrophy. Which is indicated? Hand Clin 1997; 13:
485-497.

27. Bernard JM, Macaire P. Dose-range effects of clonidine added to lidocaine for brachial plexus block. Anesthesiology 1997; 87: 277-284.

28. Forouzanfar T, Weber WE, Kemler M, van Kleef M. What is a meaningful pain reduction in patients with complex regional pain syndrome type 1? Clin J Pain 2003; 19: 281-285.

29. Carron H, Litwiller R. Stellate ganglion block. Anesth Analg 1975; 54: 567-570.

30. Elias M. Cervical sympathetic and stellate ganglion blocks. Pain Physician 2000; 3: 294-304.

31. Hardy PA, Wells JC. Extent of sympathetic blockade after stellate ganglion block with bupivacaine. Pain 1989; 36: 193196.

32. Hilgenhurst $\mathrm{G}$. The Bier block after 80 years: a historical review. Reg Anesth 1990; 15: 2-5.

33. Sia S, Lepri A. Clonidine administered as an axillary block does not affect postoperative pain when given as the sole analgesic. Anesth Analg 1999; 88: 1109-1112.

34. Eisenach JC, De Kock M, Klimscha W. Alpha(2)-adrenergic agonists for regional anesthesia. A clinical review of clonidine (1984-1995). Anesthesiology 1996; 85: 655-674.

35. Choyce A, Peng P. A systematic review of adjuncts for intravenous regional anesthesia for surgical procedures. Can $\mathrm{J}$ Anaesth 2002; 49: 32-45.

36. Reuben SS, Steinberg RB, Madabhushi L, Rosenthal E. Intravenous regional clonidine in the management of sympathetically maintained pain. Anesthesiology 1998; 89: 527530.

37. Kopacz DJ, Bernards CM. Effect of clonidine on lidocaine clearance in vivo: a microdialysis study in humans. Anesthesiology 2001; 95: 1371-1376.

38. Kroin JS, Buvanendran A, Beck DR, Topic JE, Watts DE, Tuman KJ. Clonidine prolongation of lidocaine analgesia after sciatic nerve block in rats is mediated via the hyperpolarization-activated cation current, not by alpha-adrenoreceptors. Anesthesiology 2004; 101: 488-494.

39. Hoffman BB. Cathecolamines, sympathomimetic drugs, and adrenergic receptor antagonists. In: Hardman JG, Limbird LE, Gilman AG (Editors), Goodman \& Gilman's The pharmacological basis of therapeutics. 10th edn. New York: McGraw-Hill; 2001. p 215-268.

40. Bonelli S, Conoscente F, Movilia PG, Restelli L, Francucci $B$, Grossi E. Regional intravenous guanethidine vs. stellate ganglion block in reflex sympathetic dystrophies: a randomized trial. Pain 1983; 16: 297-307. 